Results 17 requests were activated. 1 call was stood down. 15 out of the 16 requests had a JEMP delivered within 2 hours. This ranged from 34 minutes to 111 minutes. The mean time was 72 minutes.

Conclusion This is a pioneering service that developed during the coronavirus pandemic, it went from concept to a national pilot in 6 days. The uptake of services was less than originally predicted. However, it has been successful in its primary aims to maintain the supply chain and deliver vital EoL medications directly to the patient anywhere in Wales (24 hours 7 days a week) within 2 hours from the initial request. Further work needs to be done on collecting feedback and assessing sustainability.

\section{THE USE OF ONLINE VIDEOCONFERENCING FOR FOCUS GROUPS IN PALLIATIVE CARE RESEARCH}

Rebecca Gemmell, Alison Allam, Margaret Perkins, Joanne Droney. Royal Marsden NHS Foundation Trust

10.1136/spcare-2021-PCC.21

Background Qualitative focus group research traditionally involves participants meeting face-to-face- in small groups to allow for participant interaction. The Covid-19 pandemic has prevented such meetings. Consequently, research has moved to holding online meetings using videoconferencing software. We discuss the advantages and challenges of running such groups.

Method We ran two focus groups on early palliative care in haemato-oncology using the videoconferencing platform Zoom. Recruitment was conducted online using pre-existing mailing lists and in person within the hospital outpatient department. Potential participants were emailed information about the focus of the discussion,. before signing a consent form returned by post or email. Groups were audio recorded with the consent of participants and transcribed for analysis.

Results One person declined to participate due to the group's online setting. Four and eight participants respectively attended the groups, each led by a lead facilitator and two PPI co-facilitators. Participants conversed openly, using hand raising to indicate when they wanted to speak. Conversation did not appear hindered by the online setting. Participants commented that they felt able to interact easily on the virtual platform even when discussing highly emotive issues; in-depth discussion of the chosen topic was achieved. Participants thought that some participants may be deterred from participation in online videoconferencing; one person declined to participate due to the group's online setting. The virtual platform enabled participants from a wide age range and geographical location to participate. There were no significant technical challenges. One person declined to participate due to the group's online setting.

Conclusion Online videoconferencing is increasingly common in professional and social settings due to the Covid-19 pandemic. It can be used effectively in qualitative research. Choice of platform, recruitment methods, obtaining valid consent, and recording the meeting need to be carefully considered. Researchers and participants should be supported to engage with technology to support robust research but consideration must be given to several factors to ensure success.
4 RESTRICTED VISITING DURING COVID-19 PANDEMIC : AN UNCOMFORTABLE OXYMORON FOR HOSPICE STAFF

Anneka Burge, Jennifer Todd, Craig Gannon, Keetje Gull. Princess Alice Hospice

\subsection{6/spcare-2021-PCC.22}

Background Visiting in a hospice setting during COVID-19 has been incredibly emotive and challenging, not only for patients and relatives, but also for staff. Restricting visiting conflicts with holistic patient-centred care, and the complexity of discussions and decision-making cannot be underestimated, impacting on the emotional burden for staff morale and resilience.

Method A three-pronged approach was taken. 1. A hospice visiting task and finish group was set up. 2. A new role was developed, using government COVID-19 funding- a 'Visitor Co-ordinator' for an initial three-month period. This role supported decision making, communication with families and screening/practical support in the use of PPE as well as facilitating virtual visiting. 3. A 3-tiered Visitors Traffic Light Guideline (green, amber, red) providing some clarity for staff around 'essential visiting' and adapting to fluctuating local government restrictions was developed, and implemented. Feedback was collated for the visitor coordinator role, and a survey is underway to evaluate the Visitor Traffic light guideline.

Results Feedback from staff members following the introduction of the Visitor co-ordinator role was extremely positive and demonstrated an improvement in wellbeing at work. Staff reported the role of the co-ordinator relieved a 'huge burden and source of stress' and as a consequence allowed them to 'dedicate more time to patients'. The hospice visiting tiered guideline has aligned with the 'Visiting healthcare inpatient settings during the COVID-19 pandemic: principles' NHS guidelines however, some staff still report that the application of guidelines remains extremely challenging.

Conclusions A Visitor Co-ordinator role was extremely beneficial during the first weeks of the pandemic. Although helpful, a guideline alone does not alleviate all the challenges associated with restricted visiting; it remains an uncomfortable oxymoron for hospice staff and a flexible individualised risk approach is still needed to ensure compassionate visiting at the end of life.

\section{RESTRICTED VISITING AND GENDER INFLUENCE PERCEPTIONS ABOUT END-OF-LIFE CARE SUPPORT: A NATIONAL UK SURVEY OF BEREAVED RELATIVES' VIEWS}

Catriona R Mayland, Rosemary Hughes, Tamsin Mcglinchey, Warren Donnellan, Kate Bennett, Louise Dalton, Elizabeth Rapa, Steven Lane, Stephen Mason. University of Sheffield, University of Liverpool, University of Oxford

\subsection{6/spcare-2021-PCC.23}

Background The COVID-19 pandemic significantly affected experiences of death and dying for patients and families. Our focus was on bereaved relatives' perceptions about experiences of care in the last days of life during the pandemic to help inform practice and policy.

Methods A national online survey, informed by patient and public involvement, was developed and disseminated via social media, public and professional networks between June and October 2020. Validated instruments (e.g. abbreviated 'Care 
Of the Dying Evaluation' questionnaire) and purposively designed questions were used to assess the impact of COVID19. Data was analysed using descriptive statistics, logistic regression and thematic analysis of free-text responses.

Results Respondents $(n=278)$ had a mean age of 53.4 years (range 19-68); 216 (78.0\%) female and 174 (62.6\%) 'son/ daughter' to the deceased. Over half $(156,56.5 \%)$ were unable to visit during the dying phase. Almost $70 \%$ of the deceased (mean age 80.5 years; 160 (57.6\%) female) died in their 'usual place of care' (home $\mathrm{n}=30$ (10.8\%); nursing home $n=162(58.3 \%))$. This was perceived as the 'right place' by $176(75.2 \%)$ respondents. Positive perceptions were reported about nursing care compared with medical care. Unmet support was reported: 71 (30.1\%) rated emotional support as 'poor'; $110(45.5 \%)$ perceived they were inadequately supported prior to death. Being a male respondent (OR 2.9, $\mathrm{p}=0.03$ ) and able to visit during the last days of life (OR 2.2, $\mathrm{p}=0.04$ ) were independently associated with perceptions about being adequately supported. Corresponding free-text analysis suggested 'not knowing' about care was especially distressful; 'clear, compassionate leadership' contributed to positive experiences.

Conclusions Capturing these experiences during the height of the first COVID-19 wave is pertinent to inform measures to enhance care. Disconnection between dying patients and families is a key area to be addressed and the challenge of balancing individual and societal needs to enable high quality endof-life care.

\section{\begin{tabular}{|l|l}
6 & THE FIRST 6 MONTHS: WHAT DID ONE HOSPITAL
\end{tabular} SUPPORTIVE AND PALLIATIVE CARE TEAM (SPCT) EXPERIENCE DURING THE FIRST 6 MONTHS OF THE COVID-19 PANDEMIC?}

Farida Malik, Jayne Winter, David Matthews. East Sussex Healthcare NHS Trust (ESHT)

\subsection{6/spcare-2021-PCC.24}

Background 26\% of UK CoVID-19 patients requiring hospitalization die, often with high symptom burden. Needs of nonCOVID patients, however, must also be met. Studies have focused on hospital PC COVID activity but understanding the whole experience of work in the first 6 months of the pandemic is paramount to help future service planning.

Methods 1.Retrospective case series review of all patients referred to SPCT (COVID and non-Covid) 1st Mar 20-31st Aug 20 and associated service level activity. Variables collected: Baseline demographic/clinical characteristics, outcomes, Phase of Illness (POI), AKPS. Results compared with same 6-month period in 2019.

2.Semi-structured anonymised online survey of all SPCT members regarding working experiences and needs.

Results Total of 904 patient episodes: 48 COVID, 856 NonCOVID (compared with total of 826 patient episodes in 2019). Regarding 48 COVID cases, mean age 81 years, mean initial AKPS 26\%. 60\% in Dying phase, mean caseload time 4.1 days with $80 \%$ dying in hospital. SPCT involved in $30 \%$ of all COVID deaths in hospital. For Non-COVID episodes, mean age 78 years and AKPS 32\%; 27\% in Dying phase, $35 \%$ unstable, $31 \%$ deteriorating. $43 \%$ died in hospital with $24 \%$ discharged home.

For 2019 data, mean AKPS 32\% with 32\% Dying phase, $35 \%$ unstable and 21\% deteriorating.
One-third (9/12) SPC team had suspected COVID symptoms, a quarter self-isolated. Service developments included rapid introduction of 7-day CNS service \& EOL COVID guideline introduction. COVID experiences centred on rapid patient deterioration, patient communication difficulties and challenging remote communication with families. Impact on own personal wellbeing also cited. For NonCOVID, similar experiences of activity compared with preCOVID but possible later presentations highlighted and more symptomatic.

Conclusion In addition to managing COVID patients, the SPCT also saw more non-COVID patients compared with 2019. Planning for future phases underway to continue the service developments highlighted and maintain team wellbeing.

\section{COLLABORATION BETWEEN PALLIATIVE AND CRITICAL CARE: A REVIEW OF END OF LIFE CARE FOR PATIENTS WITH COVID-19 ON THE CRITICAL CARE UNIT}

Elizabeth Fleming, George Dykes, Katie Frew, David Snell. Northumbria Healthcare NHS Foundation Trust

\subsection{6/spcare-2021-PCC.25}

Introduction Providing end of life care for patients with COVID-19 on the Critical Care Unit (CCU) is complex. The novelty of disease symptomology and the impact of visiting restrictions pose significant new challenges for Palliative and Critical Care services. This review analyses end of life care for people with COVID-19 on the CCU during the first wave of the pandemic.

Methods All patients in a regional hospital who received continuous positive airway pressure (CPAP) or invasive ventilation (IV) and subsequently died on the CCU were included $(n=23)$. Data were collected through retrospective review of clinical notes.

Results 15 patients received IV. Eight received CPAP only. Two were subsequently stepped down to high flow nasal cannula (HFNC).

All patients died within 24 hours of withdrawal of respiratory support. Nine people who received IV died within one hour of treatment withdrawal.

Patients who required CPAP had a high burden of symptoms including breathlessness $(n=8)$, delirium/agitation $(n=5)$ and anxiety/fear $(n=4)$. Five patients requested removal of CPAP.

In the last 24 hours of life all eight patients on CPAP/ HFNC required an opioid. Six also required midazolam. Only two patients had syringe drivers prescribed.

All patients who were recognised as being in the last days of life had discussions documented between healthcare professionals and their relatives $(n=20)$.

Conclusions Patients treated on the CCU for COVID-19 demonstrated a high symptom burden. Time from withdrawal of ventilation to death was short. Medications required varied and the use of syringe drivers was low. This may indicate a need for earlier collaboration between Critical Care and Palliative Care teams. Despite complexities surrounding care provision in this setting there was a high standard of documented discussions with patients and families. This review highlights the need for early recognition of people being $\hat{a} €$ œsick enough to dieâ€ to ensure good quality, individualised end of life care. 